\title{
The Surface Skirt in Gaseous Scanning Electron Microscope (GSEM)
}

\section{Lahcen Khouchaf}

Ecole des Mines, Université Lille Nord de France, Douai, France.

Email: lahcen.khouchaf@mines-douai.fr

Received July $28^{\text {th }}, 2013$; revised September $17^{\text {th }}, 2013$; accepted October $9^{\text {th }}, 2013$

Copyright (C) 2013 Lahcen Khouchaf. This is an open access article distributed under the Creative Commons Attribution License, which permits unrestricted use, distribution, and reproduction in any medium, provided the original work is properly cited.

\begin{abstract}
In this work, a novel parameter called the surface skirt $\left(S_{s}\right)$ is introduced. The electron beam scattering by gaseous environment is the fundamental parameter limiting the performance of the Gaseous Scanning Electron Microscopy (GSEM). The result is the enlargement of the primary beam characterized by the radius skirt $R_{s}$. The scattering phenomena require a much closer re-examination. In fact, depending on the localization of EDX detector and the particles shape to analyze, the collected signal after the beam skirt will be different and $R_{s}$ also will be different. So, except for homogeneous materials, $R_{s}$ can not describe the scattering behavior. In this study, the surface skirt $S_{s}$ instead of the radius skirt is introduced. Unlike $R_{s}$, the results show that $S_{s}$ is a linear function versus pressure. This may help to use $S_{s}$ in different scattering regimes and for a best interpretation of the consequences of electron scattering beam by gaseous environment. For demonstration, two gas environments: helium and water vapor are given but the results are valid whatever the environment used.
\end{abstract}

Keywords: GSEM; Surface Skirt; Radius Skirt; ESEM

\section{Introduction}

Currently, Conventional Scanning Electron Microscope is working under high vacuum (about $10^{-5}$ Torr). There is much interest in introducing the gas environment in the specimen chamber which leads to the degradation of the high vacuum. At low vacuum, depending on the pressure value in the specimen chamber, different names are given in the literature such as Environmental Scanning Electron Microscope (ESEM) [1], LVSEM: Low Vacuum Scanning Electron Microscope, HPSEM: High Pressure Scanning Electron Microscope, VPSEM: Variable Pressure Scanning Electron Microscope, CPSEM: Controlled pressure Scanning Electron Microscope and depending on the maximum pressure attainable in the specimen chamber but all these microscopes differ from CSEM by the capability to introduce the gas as an environment unlike High vacuum in CSEM and the use of gaseous detection system such as Gaseous Secondary Electron Detector (GSED). Indeed, all these microscopes may be called Gaseous Scanning Electron Microscope (GSEM) [2]. The electron beam scattering by gaseous environment is the fundamental parameter limiting the performance of the Gaseous Scanning Electron Microscopy
(GSEM). The result is the enlargement of the primary beam characterized by the radius skirt $R_{s}$ [1-4].

In fact, depending on the localization of Energy Dispersive X-ray spectroscopy (EDX) detector and the particles shape to analyze, the collected signal after the beam skirt will be different and $R_{s}$ also will be different whatever the formula used. So, except for homogeneous materials, $R_{s}$ cannot explain or describe alone what happen. Several works have been performed but the scattering phenomena require a substantial revision [5-16].

In this study, the use of surface skirt $S_{s}$ instead of the radius skirt is suggested. Examples are given with two gas environments: helium and water vapor.

\section{Results and Discussion}

An accelerating voltage of $20 \mathrm{keV}$ and a total gas path length of $2 \mathrm{~mm}$ (the standard conditions used in GSEM) using the long detector for microanalysis and two gases: $\mathrm{H}_{2} \mathrm{O}$ and $\mathrm{He}$ are used as conditions.

Elastic and inelastic scattering take place after the interaction between the primary electron beam and the atoms or the molecules of the gas present in the specimen chamber. Recent experimental and theoretical measure- 
ments of elastic, inelastic and total electron cross section are available [17-19]. The inelastic scattering produces the generation of characteristic and continuum X-rays and the elastic scattering produces a deviation of the primary electron beam by forming a skirt around the focused probe (Figure 1).

The average collision number with particle gas per electron is given by the equation below:

$$
m=\sigma_{t} \times n \times L
$$

where $\sigma_{t}$ : scattering cross section is specific to each gas molecule; $n$ : gas particle number/volume; $L$ : Working distance (distance between the final aperture PLA1 and the surface of the sample see Figure 1). The Gas Path Length GPL is introduced and corresponds to the distance that electrons have to travel through the gas to reach the sample. $m$ may be expressed as below [1]:

$$
m=\frac{\sigma_{T} L P}{k T}
$$

where $\sigma_{T}$ is the total cross section of the gas, $L$ the gas path length, $P$ the gas pressure, $k$ the Boltzmann constant and $T$ the temperature. Depending on the value of $m$ it is possible to define three different scattering regimes. Elastic scattering leads to the enlargement of the primary electron beam to form a skirt producing the generation of $\mathrm{X}$-rays which are not representative of the zone of interest for X-ray microanalysis. Different correction methods have been developed in order to take into account the contribution of the skirt. Up to now these methods have not been successful. Adapted from Reimer [3], Danilatos [1] introduced the radius $R_{s}$ (Figure 1) which represents the radius containing $90 \%$ of the incident beam as below:

$$
R_{s}=\frac{364 Z}{E} \cdot\left(\frac{P}{T}\right)^{1 / 2} \cdot L^{3 / 2}
$$

where $R_{s}$ is the skirt radius, $Z$ the gas atomic number, $\mathrm{E}$ the incident beam energy, $P$ the pressure, $T$ the temperature and L corresponds to the gas path length (GPL).

Currently the radius of the skirt is used to describe the

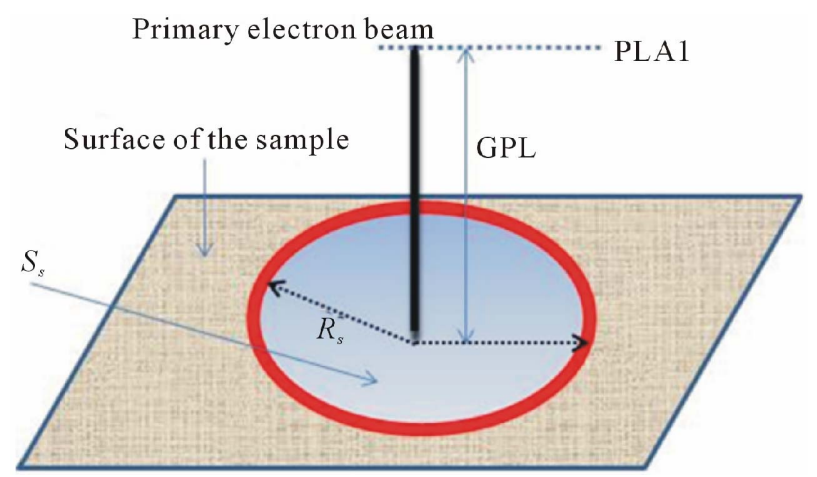

Figure 1. Description of the radius $R_{s}$ and the surface $S_{s}$ of the skirt. consequences of the electron beam scattering by the gas on the resolution in imaging and microanalysis [5-14]. The Equation (3) of $R_{s}$ given above is one of the most used expressions to follow this phenomenon. Figure 2 shows the evolution of $R_{s}$ versus the pressure for two gases: water vapor and helium.

For water vapor, we can notice the same beahvior (nonlinear) of $R_{s}$. This means that at low energy (not presented here), the working pressure must be low in order to keep acceptable conditions of resolution. For helium, the nonlinear appearance remains the same at low and high energy with increase of the $R_{s}$ values. On the other hand, the values of $R_{s}$ are very low compared to water vapor. For helium, the condition of resolution at low energy is almost the same that for water vapor at high energy. Considering scattering mode both gases have the same behavior.

Depending on the localization of EDS detector and the particles shape to analyze, the collected signal coming from the beam skirt will be different and $R_{s}$ also will be different. Except for homogeneous materials, $R_{s}$ cannot explain or describe what happens during electron scattering by gases. Hence, some additional development should be performed in order to have an accurate description the contribution of the beam skirt (Figure 1). The impact of the scattering electron on the surface of the sample forms a disk. if the radius of the unscattered beam is neglected in comparison with the scattered beam as the first approximation. In order to take into account this approach we introduced a surface of the skirt $S_{s}$ instead of the $R_{s}$. In the case $S_{s}$ is given by the equation below:

$$
\begin{gathered}
S_{s}=\pi \cdot r^{2} S \\
S_{s}=\pi \cdot\left(\frac{364 Z}{E}\right)^{2} \cdot \frac{P}{T} \cdot \mathrm{L}^{3} \\
S_{s}=\pi \cdot\left(\left(\frac{364 . Z}{E}\right)^{2} \cdot \frac{L^{3}}{T}\right) \cdot P
\end{gathered}
$$

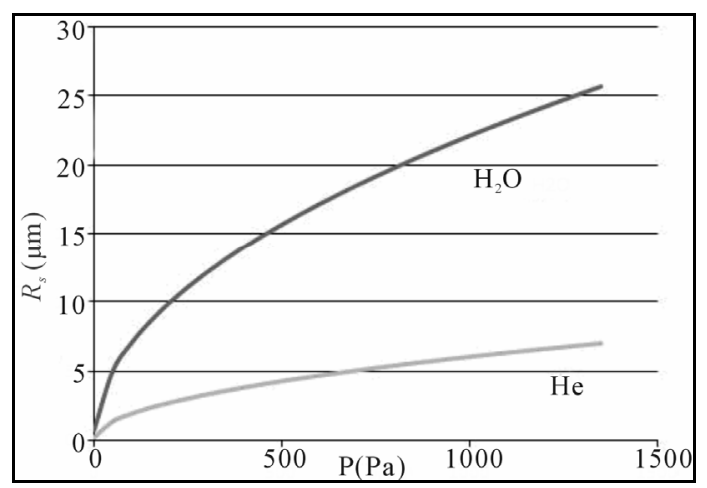

Figure 2. Variation of $R_{s}$ versus pressure at $20 \mathrm{keV}$ and $\mathrm{GPL}=2 \mathrm{~mm}$ for (a) $\mathrm{H}_{2} \mathrm{O}$ vapor; (b) He. 


$$
S_{s}=\alpha \cdot P
$$

Unlike $R_{s}$ expression given above, the Equation (6) shows that $S_{s}$ is a linear function versus the pressure. Figure 3 shows the evolution of $S_{s}$ versus the pressure for water vapor and helium.

For water vapor, at high and low energy it may be seen that $S_{s}$ increases linearly as a function of pressure. As expected, at constant pressure the values of $S_{s}$ are very important at low energy compared to that at high energy. This means that at low energy, the working pressure must be low in order to keep acceptable conditions of resolution.

For helium, at high and low energy it may be seen that $S_{S}$ increases linearly as a function of pressure. Also as expected, at constant pressure the values of $S_{s}$ are very important at low energy compared to that at high energy. This means that at low energy, the working pressure must be low in order to keep acceptable conditions of resolution i.e. to work under conditions of single scattering mode.

Therefore, unlike $R_{s}, S_{s}$ varies linearly with pressure. For two values of pressure, the measurement of $S_{s}$ leads to draw the curve $S_{s}$ versus $P\left(S_{s}=\alpha \cdot P\right)$ and thus determine the slope $\alpha$. It becomes possible to predict for any value of the pressure the surface of the area that will contribute to the EDS spectrum and thus estimate the resolution of the $\mathrm{X}$ ray microanalysis. The experimental measurement of $S_{s}$ is not a simple thing because there are many signals contributions to isolate before. We are working in order to build a new facility to measure different signals emitted after the impact of the electron beam on the sample surface.

\section{Conclusion}

Except for homogeneous materials, the radius skirt $R_{S}$ usually used in different studies can not describe the scattering behavior in GSEM. In this work a novel parameter called the surface skirt $\left(S_{S}\right)$ is introduced. The advantage of $S_{s}$ is the linearity versus the pressure. Unlike $R_{s}$, the curve Ss versus the pressure is independent of the scattering regimes. For illustration, two gas

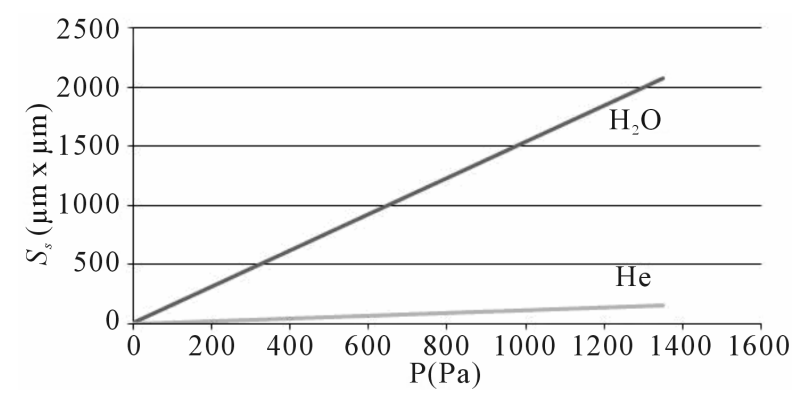

Figure 3. Variation of $S_{s}$ versus pressure at $20 \mathrm{keV}$ and WD $=2 \mathrm{~mm}$ for (a) $\mathrm{H}_{2} \mathrm{O}$ vapor; (b) $\mathrm{He}$. environments: helium and water vapor are used.

\section{REFERENCES}

[1] G. D Danilatos, "Foundations of Environmental Scanning Electron Microscopy," Advances in Electronics and Electron Physics, 71, 1988, pp. 109-250.

http://dx.doi.org/10.1016/S0065-2539(08)60902-6

[2] L. Khouchaf, "Gaseous Scanning Electron Microscope (GSEM): Applications and Improvement," In: V. Kazmiruk, Ed., Scanning Electron Microscopy, InTech, Croatia, 2012, pp. 1-16. http://dx.doi.org/10.5772/34930

[3] L. Reimer, "Scanning Electron Microscopy," SpringerVerlag, Berlin, Heidelberg, New York, Tokyo, 1985.

[4] D. Stokes, "Principles and Practice of Variable Pressure: Environmental Scanning Electron Microscopy (VP-ESEM)," Wiley, Hoboken, 2008. http://dx.doi.org/10.1002/9780470758731

[5] J. F. Mansfield, "X-Ray Microanalysis in the Environmental SEM: A Challenge or a Contradiction?" Microchimica Acta, Vol. 132, No. 2, 2000, pp. 137-143. http://dx.doi.org/10.1007/s006040050054

[6] C. Gilpin and D. C. Sigee, "X-Ray Microanalysis of Wet Biological Specimens in the Environmental Scanning Electron Microscope. 1. Reduction of Specimen Distance under Different Atmospheric Conditions," Journal of Microscopy, Vol. 179, No. 1, 1995, pp. 22-28. http://dx.doi.org/10.1111/j.1365-2818.1995.tb03610.x

[7] R. Gauvin, "Some Theoretical Considerations on X-Ray Microanalysis in the Environmental or Variable Pressure Scanning Electron Microscope," Scanning, Vol. 21, No. 6, 1999, pp. 388-393. http://dx.doi.org/10.1002/sca.4950210605

[8] L. Khouchaf and J. Verstraete, "X-Ray Microanalysis in the Environmental Scanning Electron Microscope (ESEM): Small Size Particles Analysis Limits," Journal de Physique IV, Vol. 12, No. 6, 2002, pp. 341-346. http://dx.doi.org/10.1051/jp4:20020242

[9] L. Khouchaf and J. Verstraete, "Electron Scattering by Gas in the Environmental Scanning Electron Microscope (ESEM): Effects on the Image Quality and on the X-Ray Microanalysis," Journal de Physique IV, Vol. 118, 2004, pp. 237-243. http://dx.doi.org/10.1051/jp4:2004118028

[10] L. Khouchaf and F. Boinski, "Environmental Scanning Electron Microscope study of $\mathrm{SiO}_{2}$ Heterogeneous Material with Helium and Water Vapor," Vacuum, Vol. 81, No. 5, 2007, pp. 599-603. http://dx.doi.org/10.1016/j.vacuum.2006.08.001

[11] O. Mansour, K. Aidaoui, A. Kadoun, L. Khouchaf and C. Mathieu, "Monte Carlo Simulation of the Electron Beam Scattering under Gas Mixtures Environment in an HPSEM at Low Energy," Vacuum, Vol. 84, No. 4, 2010, pp. 458-463. http://dx.doi.org/10.1016/j.vacuum.2009.09.004

[12] L. Khouchaf, C. Mathieu and K. Abd-Ed-Daïm, "Microanalysis Results with Low Z Gas inside Environmental SEM," Vacuum, Vol. 86, No. 1, 2011, pp. 62-65. http://dx.doi.org/10.1016/j.vacuum.2011.04.014

[13] C. Arnoult, J. Di Martino, L. Khouchaf, V. Toniazzo and 
D. Ruch, "Pressure and Scattering Regime Influence on the EDS Profile Resolution at a Composite Interface in Environmental SEM," Micron, Vol. 42, No. 8, 2011, pp. 877-883. http://dx.doi.org/10.1016/j.micron.2011.06.004

[14] A. Zoukel, L. Khouchaf, J. D. Martino and D. Ruch, "Skirting Effects in the Variable Pressure Scanning Electron Microscope: Limitations and Improvements," $\mathrm{Mi}$ cron, Vol. 44, 2013, pp. 107-114. http://dx.doi.org/10.1016/j.micron.2012.05.004

[15] A. Zoukel, L. Khouchaf, C. Arnoult, J. D. Martino and D. Ruch, "A New Approach to Reach the Best Resolution of X-Ray Microanalysis in the Variable Pressure SEM," Micron, Vol. 46, 2013, pp. 12-21. http://dx.doi.org/10.1016/j.micron.2012.11.003

[16] O. Mansour, A. Kadoun, L. Khouchaf and C. Mathieu, "Monte Carlo Simulation of the Electron Beam Scattering under Water Vapor Environment at Low Energy," Vacuum, Vol. 87, 2013, pp. 11-15.

http://dx.doi.org/10.1016/j.vacuum.2012.06.006

[17] J. Rattenberger, J. Wagner, H. Schrottner, S. Mitsche and A. Zankel, "A Method to Measure the Total Scattering Cross Section and EffectiveBeam Gas Path Length in a Low-Vacuum SEM," Scanning, Vol. 31, No. 3, 2009, pp. 107-113. http://dx.doi.org/10.1002/sca.20148

[18] S. A. Wight and A. R. Konicek, "Electron Scattering Cross Section Measurements in a Variable Pressure Scanning Electron Microscope," Micron, Vol. 43, No. 9, 2012, pp. $985-991$. http://dx.doi.org/10.1016/j.micron.2012.04.003

[19] G. D. Danilatos, "Electron Scattering Cross-Section Measurements in ESEM," Micron, Vol. 45, 2013, pp. 1-16. http://dx.doi.org/10.1016/j.micron.2012.10.002 